\title{
Introduction: complementary medicine in ophthalmology
}

\author{
Lori Ventura
}

Received: 20 February 2009 / Accepted: 9 July 2009/Published online: 5 August 2009

(C) The Author(s) 2009. This article is published with open access at Springerlink.com

Keywords Complementary medicine .

Psychoneuroimmunology $\cdot$ PNI $\cdot$ Cytokines $\cdot$ Immune system $\cdot$ Neuroprotection $\cdot$ Moxibustion $\cdot$ Lei-huo-jiu therapy · Acupuncture $\cdot$ Wolfberry Chuanxiong ·

Tetramethylpyrazine $\cdot$ Cistanche tubelosa $\cdot$ Bear bile .

Traditional Chinese medicine $\cdot$ TCM $\cdot$ Macular

degeneration $\cdot$ Retinal degeneration $\cdot$ Glaucoma $\cdot$ Keratitis

sicca $\cdot$ Dry eye

International Congress of Eye Research (ICER)'s September 2008 meeting in Beijing provided a unique conference of East meets West on healing approaches in ophthalmology, and for the first time, a section on complementary medicine (CM) was added to the program. CM includes all fields of healing including psychotherapy, hypnosis, meditation and visual imagery, prayer, acupuncture, herbal remedies and nutrients, massage therapy, exercise, reiki, and other measures which may facilitate healing other than traditional Western medicines and procedures. Before a therapy gains global acceptance and use, it must withstand the rigor of scientific investigation and pass through the narrow straits of peer review and possibly Food and Drug Administration approval to render it safe and effective. ICER's goal was to encourage investigators of complementary methodology to cross that threshold. To that end, three $\mathrm{CM}$ sessions included 21 presentations which introduced novel complementary therapeutic approaches in the treatment of ocular conditions. This monograph has been designed to feature some of those presentations.

\section{Ventura $(\square)$}

University of Miami Miller School of Medicine,

Bascom Palmer Eye Institute,

Miami 33136 FL, USA

e-mail: lventura@med.miami.edu
Immunological mechanisms have been clearly established at the core of a number of conditions to include not only cancer and coronary artery disease but also several ocular conditions such as uveitis, thyroid eye disease, keratitis sicca, ocular infections, and more recently for macular degeneration and glaucoma. Four investigators have focused on immune mechanisms in ocular disease. Chun Zhang from Peking University Eye Center presented his group's work which explored the balance of Th1 and Th2 cytokines in glaucoma. Zhang's group showed altered levels of cytokines in mild glaucomatous neuropathy. They suggest the possibility that abnormal immune environments contribute to the pathophysiology of glaucoma.

The second group, lead by Michal Schwartz, has pioneered the concept of harnessing the immune system to combat neurodegeneration in glaucoma. They have shown that immune deficiency or suppression impair the recovery process after optic nerve crush, whereas boosting selfspecific immune response, by both passive and active immunization, promotes recovery. Those same $\mathrm{T}$ cells that can lead to the development of autoimmune disease can protect neurons under neurodegenerative conditions, and it is a subpopulation of regulatory $\mathrm{T}$ cells which regulates the autoimmune response to promote protection over injury. Dr. Schwartz introduces the concept of a therapeutic vaccination to boost the immune response to facilitate neuroprotection.

This delicate balance of immune protection over injury may be overridden by physical or emotional stressors. In 1975, from the groundbreaking work of Robert Ader [1] at New York's University of Rochester, the field of psychoneuroimmunology (PNI) was born and has since become a burgeoning arena in neuroscience research. PNI is a discipline which describes the relationship between emotional states, the central and peripheral nervous systems, and the endocrine and immune systems. The theoretical consequence of that link is that negative psychological 
experiences, such as stress and anxiety, can influence immune function, which in turn may have an effect on the course of disease. A number of medical conditions including HIV infection, cancer, and autoimmune diseases have been studied using animal models as well as clinically to document the negative effects of stress on physical disease states. A comprehensive review of clinical research in this field can be found in the text Human Psychoneuroimmunology [2] by Dr. Kavita Vedhara from the University of Bristol and Michael Irwin, M.D. of the Cousin's Center for Psychoneuroimmunology at University of California, Los Angeles. In this monograph, I present a historical review of how physicians have deviated from a more holistic approach to healing over time. My article focuses on pathophysiological mechanisms of stress and how these mechanisms may impact ocular disease states.

Keratitis sicca was investigated by Ming Jing with a mouse model to investigate Chinese herbs, and a clinical study led by Namtran Pham studied the effects of acupuncture for dry eye. Jing worked with Lei-huo-jiu, which is made up of 30 types of herbs delivered using a moxibustion technique over both eyes in humans. Lei-huo-jiu is thought to adjust ocular perfusion and its effect is thought to be similar to that of acupuncture. Using a nonobese diabetic mouse model of Sjogren's syndrome, Ming Jing reported the benefits of Lei-huo-jiu therapy alone vs combination with herbal therapy. In the mouse, a smoking box was devised to deliver the agents. The lacrimal gland was excised and histological as well as immunohistochemical studies were performed using Image Plus Pro to afford a quantitative measure of interleukin-1, tumor necrosis factor alpha-1, and nuclear factor kappa B in the specimens. She showed reduction of all three cytokines in the combined therapy group compared to controls.

Lei-huo-jiu therapy has been described to have effects that are similar to those of acupuncture, and it was shown to be efficacious in this animal model of keratitis sicca as presented by Jing. Empirical study of Lei-huo-jiu therapy in the Chinese literature has been touted to enhance circulation rapidly. Acupuncture has been proposed to enhance parasympathetic tone in the various meridia where it is applied. The relationship between Lei-huo-jiu therapy and acupuncture may be that they are both enhancers of parasympathetic tone. One may ask why would this be beneficial for the dry eye? A recent publication by Liu et al. [3] confirmed the presence of all five subtypes of muscarinic cholinergic receptors in human corneal limbal and conjunctival epithelial cells, conjunctival goblet cells, and corneal endothelial cells. Not only are the muscarinic receptor subtypes widely distributed in corneal and conjunctival tissues but their natural ligand, acetylcholine, is also abundant on the ocular surface - secreted by parasympathetic neurons and ocular surface epithelial cells [4]. Furthermore, Liu showed that activation of muscarinic receptors by carbachol led to increased conjunctival epithelial cell proliferation [3]. The discovery of corneal muscarinic receptors was provocative as a possible mechanism of action of how acupuncture or Lei-huo-jiu therapy may favorably impact the ocular surface in the study presented here by Jin Ming.

Embracing well-established disease models to conduct carefully controlled studies, four investigators suggested a promising prospect for using naturopathic compounds in the treatment of retinal neurodegenerative diseases. Wolfberry-commercially called goji berry-is the common name for the fruit of two very closely related species Lycium barbarum and Lycium chinense. Renowned in Asia as a highly nutritious food, wolfberries have been used in traditional Chinese medicine to improve vision for more than 2,500 years. Since the early twenty-first century in the USA and other such developed countries, there has been rapidly growing recognition of wolfberries for their nutrient value and antioxidant qualities, leading to a profusion of consumer products. Such rapid commercial development extends from wolfberry having a high ranking among super fruits [5], and it is expected to be part of a multibillion dollar market by $2011[6,7]$. A clinical study by Amagase and Nance in 2008 showed that the daily consumption of $L$. barbarum delivered in a standardized juice preparation for 14 days increases subjective feelings of general well-being and improves neurological/psychological performance and gastrointestinal functions [8]. L. barbarum polysaccharides as a food supplement have been shown in several studies to have a complex range of immunomodulatory effects. Kwok-Fai So's group evaluated the modulation of $L$. barbarum polysaccharides on retinal microglia and its neuroprotective effect on the survival of retinal ganglion cells (RGCs) using a rat model of chronic ocular hypertension. He showed that oral administration of $L$. barbarum in Sprague-Dawley rats (250-280 g) significantly reduced the loss of RGCs in the face of elevated intraocular pressure.

For hundreds of years, Chinese physicians have used chuanxiong for treatment of stroke and heart and kidney conditions [9]. Chuanxiong is a frequently used Chinese herb, also called ligustrazine, which has the chemical name tetramethylpyrazine (TMP). Studies indicate that TMP treatments improve cardiac and cerebral blood flow and enhance perfusion of the nail bed microcirculation $[10,11]$. Working with TMP, Zhiqun Tan fed both 10 - and 16-month-old 3xTg Alzheimer's transgenic mice a diet containing TMP $(300 \mathrm{mg} / \mathrm{kg}$ diet $)$ for 2 months. Tan showed significant amelioration of the Alzheimer's pathological changes in both brain and retina with TMP treatment. Tan cites several studies which imply that TMP 
has exhibited a calcium antagonist role in vascular tissues, functions as an ROS scavenger, and inhibits inflammatory events in vivo possibly through modulating secretion of specific cytokines and nitric oxide-related pathways.

Use of extracts of Cistanche tubelosa for eye disease has been documented as early as 1644 in the Shenshi Yaohan by Fu Renyu where it is an important part of a treatment for several conditions that may include glaucoma and macular degeneration. Colin Barnstable tested extracts of $C$. tubelosa on retinal ganglion cell line RGC5. The extracts increased mitochondrial membrane potential and resulted in an increased expression of a several genes, including a number coding for mitochondrial proteins that include subunits of ATP synthase, citrate synthase, and SOD1. Barnstable has shown that the survival of retinal neurons under a variety of stresses can be dramatically improved by a number of dietary compounds which have effects on mitochondria.

Bear bile has been included in Asian pharmacopoeias for thousands of years in the treatment of several diseases. Tauroursodeoxycholic acid (TUDCA) and ursodeoxycholic acid are the major hydrophilic bile acids of bear bile. Both of these are available as synthetic formulations and are approved by the health administrations of several countries for treatment of cirrhosis and gallstones. Jeffrey Boatright, in an elegant study, showed that treatment with TUDCA is protective in whole animal and cell culture models of retinal degeneration.

The fine efforts of these investigators have been published here to showcase how the door to scientific scrutiny has been flung open to the realm of complementary medicine in ophthalmology to invite others in vision research to follow suit.
Acknowledgement NIH center grant P30-EY014801, unrestricted grant to Bascom Palmer Eye Institute from Research to Prevent Blindness.

Open Access This article is distributed under the terms of the Creative Commons Attribution Noncommercial License which permits any noncommercial use, distribution, and reproduction in any medium, provided the original author(s) and source are credited.

\section{References}

1. Ader R, Cohen N. Behaviorally conditioned immunosuppression. Pschosom Med. 1975;37(4):333-40.

2. Vedhara K, Irwin M. Human psychoneuroimmunology. Oxford: Oxford University Press; 2005.

3. Liu S, Li J, Tan D, Beuerman R. Expression and function of muscarinic receptor subtypes on human cornea and conjunctiva. IOVS. 2007;48:2987-96.

4. Nietgen GW, Schmidt J, Hesse L, Honemann CW, Durieux ME. Muscarinic receptor functioning and distribution in the eye: molecular basis and implications for clinical diagnosis and therapy. Eye. 1999;13:285-300.

5. Sohn E. Superfruits, superpowers? Los Angeles Times, March 10, 2008

6. McNally A. Superfruits marked set to double by 2011 Nutraingredients.com-Europe October 8, 2007

7. Runestad T. Functional ingredients market overview, Functional Ingredients, October, 2007

8. Amagase H, Nance DM. A randomized, double-blind, placebocontrolled study of the general effects of a standardized Lycium barbarum (Goji) juice, GoChi ${ }^{\mathrm{TM}}$. J Altern Complement Med. 2008;14(4):403-12.

9. Cai Y, Ren M, Yang R. Observation on curative effect of acute ischemic cerebrovascular disease treated with different dosage of ligustrazine. Chin J Integr Tradit West Med. 2000;20(10):747-9.

10. Yan F, Luo R. Effects of ligustrazine on blood vessels and blood components. Zhong Yao Cai. 2002;25:143-5.

11. Wang GJ. The change in nailfold microcirculation in patients with acute cerebral thrombosis treated with ligustrazine. Zhonghua Shen Jing Jing Shen Ke Za Zhi. 1984;17:121-4. 